\title{
USING RESISTIVITY TO MEASURE H/PD AND D/PD LOADING: METHOD AND SIGNIFICANCE
}

\author{
M.C.H. MCKUBRE \\ SRI International, Materials Research Laboratory, 333 Ravenswood Avenue \\ Menlo Park, CA 94025/USA \\ F.L. TANZELLA \\ SRI International, Materials Research Laboratory, 333 Ravenswood Avenue \\ Menlo Park, CA 94025/USA
}

\begin{abstract}
The resistance ratio method is the most frequent technique used to determine the extent of interstitial loading of hydrogen or deuterium atoms into palladium electrodes, or extended structures used in electrolytic or gas phase cold fusion experiments. Specifically, advantage is taken of an empirical relationship between the measured resistance, $R$, normalized to that of the same body at the same temperature in the absence of hydrogen isotope, $\mathrm{R}^{\circ}$, hence $\mathrm{R} / \mathrm{R}^{\circ}$, and the atomic fraction occupancy of octahedral interstitials, $\mathrm{x}=\mathrm{H} / \mathrm{Pd}$ or $\mathrm{D} / \mathrm{Pd}$. This method was first suggested and employed in cold fusion studies by the present authors [1], and received immediate and widespread acceptance because of the ease with which this experimental technique could be used to make insitu, real-time measurements of a parameter, $\mathrm{D} / \mathrm{Pd}$, anticipated [2] or hypothesized [3-6] at that time to relate to cold fusion heat excess or nuclear production.

We take up this topic again 15 years later in an attempt to clear up some errors and misunderstandings regarding the resistance ratio method and its application in cold fusion studies. The relationship between $\mathrm{R} / \mathrm{R}^{\circ}$ and $\mathrm{x}$ is empirical. That is, calibrations are only as good as the experiments that support the shape of the curve and cannot be used outside the range $[\mathrm{P}, \mathrm{T}, \mathrm{x}]$ in which data are taken. The original calibration [1] (unaccountably and erroneously immortalized as the "famous Baranowski curve") involved an extrapolation of known data into the region of cold fusion interest in the D-Pd system, at $\mathrm{x}>0.6$.

Present theory and results focus new attention on the very high loading region as $\mathrm{x}$ approaches or even exceeds unity, where double occupation of octahedral sites, tetrahedral site occupancy, new phase formation or new electrical states, may be relevant to the underlying physical process of excess heat and nuclear production. Rather than simply using the resistance ratio as a qualitative tool to determine whether an electrode is better or lesser loaded, it is now important to obtain accurate quantitative information for $\mathrm{x}$ close to unity. With further experimentation and analysis of published data it is apparent that the curve originally published in 1990 is in error in the high loading condition. This paper describes how this empirical fit has been improved over the years for both $\mathrm{H} / \mathrm{Pd}$ and D/Pd by employing new data, new analysis of old data, new experimental methods and results.
\end{abstract}

\section{Introduction}

The first paper drawing attention to resistance ratio measurement as a means to quantify deuterium loading in Fleischmann-Pons electrolytic cold fusion experiments was presented by the present authors at the First Annual Cold Fusion Conference held in Salt Lake City in March 1990, and publish in the ensuing proceeding [1]. The authors of that paper made three introductory comments:

None of the "cold fusion" electrolysis experiments described to data contain any means of determining the $\mathrm{D} / \mathrm{Pd}$ content in-situ. Yet this ratio may be a crucial difference between those experiments that have produced a Fleischmann-Pons effect and those that have not.

The resistance of $\mathrm{Pd}$ metal is a function of its hydrogen content [7] and is in principle, the easiest way of determining the state of the Pd electrode as the experiment proceeds.

The relationship between the resistance and the $\mathrm{D} / \mathrm{Pd}$ ratio is known only up to 0.65 . Comparison can be made with the $\mathrm{H} / \mathrm{Pd}$ system which is calibrated in resistance up to $\mathrm{H} / \mathrm{Pd}=1.1$ [8].

The first two comments proved to be prophetic. A clear distinction can be made on the basis of average $\mathrm{D} / \mathrm{Pd}$ loading measured by resistance ratio between successful and unsuccessful excess heat production in electrolytic Fleischmann-Pons experiments. Resistance ratio measurements quickly became and remain the most widely used method of determining loading in-situ.

\section{Experimental}

In 1990 the resistance versus composition function had been characterized for the light hydrogen system up to $\mathrm{H} / \mathrm{Pd}=1.1$ [8]. This function was less well specified for $\mathrm{D} / \mathrm{Pd}$. Our initial attempt to estimate the curve based on known data [9] up to $\mathrm{D} / \mathrm{Pd}=0.65$ combined with the assertion that the resistance ratio maxima would occur at the same composition of $\mathrm{H}$ and $\mathrm{D}$, resulted in the somewhat distorted curve presented at ACCF1 [1] and replotted here as Figure 1. The fourth order polynomial extrapolation plotted as a grey line in Figure 1 formed the basis of all early estimates of $\mathrm{D} / \mathrm{Pd}$ loading from resistance ratio measurements. 


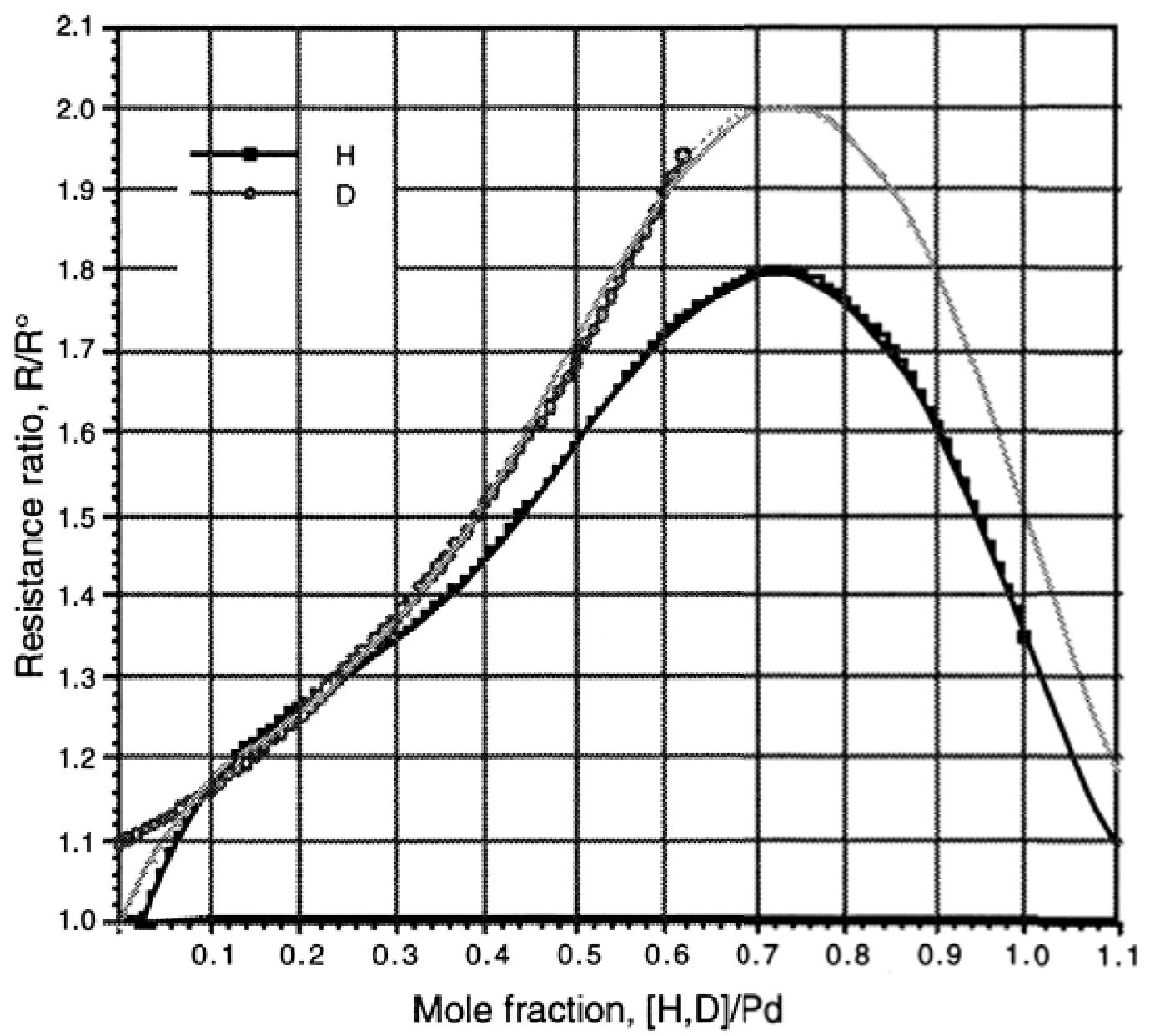

Figure 1 Original calibration curves [1].

Between 1990 and 1993 a campaign of measurements was undertaken to refine the calibration of the resistance curve for $\mathrm{D} / \mathrm{Pd}$. A number of methods were used to measure composition independent of resistance in the $\mathrm{D} / \mathrm{Pd}$ system, each with important advantages and disadvantages. Some of these methods are briefly reviewed here:

I. Macro-gravimetric. The most direct method to determine the extent of absorbed hydrogen isotope is to weigh the cathode after prolonged loading. Both the resistance and mass measurement can be performed ex-situ after washing and drying the electrode. The primary disadvantages being relatively low accuracy because of systematic errors due to the presence of surface adsorbed nonhydrogen species and absorbed but non-interstitial hydrogen, and the lack of access to the high loading region because of rapid deloading.

II. Micro-gravimetric. An in-situ gravimetric measurement was reported by NRL also at ACCF1[2]. Cheek and O'Grady used a quartz crystal microbalance to measure the mass of a deposited palladium surface film operated as a cathode. A critical difficulty with this method is the lack of access to the high loading region due to buoyancy effects associated with the presence of internal $\mathrm{D}_{2}$-filled voids and surface attached $\mathrm{D}_{2}$ bubbles at current densities sufficient to produce high loading. This method is also affected by roughening and spallation of the Pd film from the quartz support at high loading and has seldom been used.

III. Dilatometry. A number of people but notably Storms [10] took advantage of the lattice expansion that occurs when hydrogen isotopes occupy the octahedral interstitial sites in the fcc Pd lattice.

Either linear extension or volumetric expansion measurements can be used to estimate the extent of hydrogen isotope absorption provided this is wholly interstitial. A very important outcome of the extensive work by Storms using the technique was that a significant fraction of the hydrogen or 
deuterium atoms absorbed by palladium recombine in closed voids within the metal causing compressive stress and volume expansion greater than that attributable to the change in lattice parameter. Storms called this parameter "free volume". Electrodes with a large fraction of free volume tend to load poorly and to not produce excess heat [10].

IV. Oxygen displacement in closed cells. The equations that govern the cathodic absorption of H (or D) in thermodynamically closed cells can be written for basic light water electrolytes as:

$\begin{array}{ll}\text { Cathode: } & \mathrm{H}_{2} \mathrm{O}+\mathrm{e}^{-} \Rightarrow \mathrm{OH}^{-}+\mathrm{H}_{\mathrm{ads}} \\ \text { Cathode recombination } & \mathrm{H}_{\mathrm{ads}}+\mathrm{H}_{\mathrm{ads}} \Rightarrow \mathrm{H}_{2} \\ \text { Cathode absorption: } & \mathrm{H}_{\mathrm{ads}} \Rightarrow \mathrm{H}_{\mathrm{abs}} \\ \text { Anode: } & 2 \mathrm{OH}^{-} \Rightarrow \mathrm{H}_{2} \mathrm{O}+2 \mathrm{e}^{-}+\mathrm{O}_{\text {ads }} \\ \text { Anode recombination: } & \mathrm{O}_{\mathrm{ads}}+\mathrm{O}_{\mathrm{ads}} \Rightarrow \mathrm{O}_{2} \\ \text { Molecular recombination: } & 2 \mathrm{H}_{2}+\mathrm{O}_{2} \Rightarrow 2 \mathrm{H}_{2} \mathrm{O}\end{array}$

In the absence of reaction (2b) the rates of production and recombination of $\mathrm{H}_{2}$ and $\mathrm{O}_{2}$ are stoichiometrically balanced. Hydrogen occluded in the cathode by reaction (2b) is not available to recombine to water on the catalyst surface (5), resulting in a net production of $\mathrm{O}_{2}$ gas. This gas can be measured as a pressure increase (at constant volume, $\mathrm{V}$, and temperature, $\mathrm{T}$ ) or a volume increase (at constant pressure, $\mathrm{P}$, and T). One favored method is simply to displace an inert liquid onto a balance to be weighed.

In the presence of an ideally performing catalyst this method is easily employed and relatively reliable. As is true for most of the methods discussed here, it is not capable of distinguishing between interstitial absorption and hydrogen occluded in free volumes within the Pd bulk. This method was widely used by Riley et. al., at the National Cold Fusion Institute (NCFI) [11]. The data obtained from their studies were used to refine the SRI resistance ratio vs. loading curve.

V. Hydrogen displacement in closed cells. In general recombination catalysts operate more reliably at gas concentration ratios well away from the stoichiometry point $\left(2: 1 \mathrm{H}_{2}: \mathrm{O}_{2}\right)$. Method IV also has the disadvantage of making the environing and dissolved gas concentrations oxygen rich, thus lowering the $\mathrm{H}_{2}\left(\right.$ or $\left.\mathrm{D}_{2}\right)$ partial pressure and reducing the maximum loading. A method was developed at SRI [12] to precharge sealed and thermodynamically closed electrolytic cells with a controlled atmosphere of hydrogen gas. In this situation the net loading that results from equation $(2 b)$ reduces the moles of $\mathrm{H}_{2}\left(\right.$ or $\left.\mathrm{D}_{2}\right)$ in the gas phase. This can be readily measured as a pressure change (at constant $\mathrm{T}$ and $\mathrm{V}$ ) or volume change (at constant $\mathrm{T}$ and $\mathrm{P}$ ).

VI. Stripping coulometry. An anodic reaction process involving successively the reverse of reactions ( $2 \mathrm{~b}$ ) and (1) can be used very accurately to titrate loaded H (or D) from the lattice provided that the surface atom recombination reaction (2a) is avoided. This reaction can be effectively poisoned by electrodepositing heavy metals on the cathode surface. Since this also prevents absorption the codeposition of metals such as $\mathrm{Hg}$ is best done at or near the end of hydrogen loading. A procedure of this type was developed for $\mathrm{H}$ and $\mathrm{D}$ loading of long thin Pd wires by Celani 's group at INFN Frascati [13]. This method was further developed collaboratively by Tripodi at SRI for the purpose of measuring temperature effects on resistance [14].

VII. X-ray diffraction. The position of $\mathrm{Pd}$ atoms in their sublattice of $\mathrm{PdH}_{\mathrm{x}}$ or $\mathrm{PdD}_{\mathrm{x}}$ is a function of the loading, $\mathrm{x}$. Although reasonably well established for $\mathrm{PdH}_{\mathrm{x}}$, particularly for $\mathrm{x} \leq 0.85$, no reliable information exists for the condition of interest in cold fusion experiments, $\mathrm{PdD}_{\mathrm{x}}$ for $\mathrm{x}>0.85$. A collaboration between the groups at the U.S. Naval Research Laboratory (NRL) and ENEA, Frascati in Italy has been established to redress this deficiency [15]. As well as the expected increase in lattice parameter with increasing octahedral occupation, the researchers are also interested in pursuing evidence for tetrahedral site occupation, double occupation of octahedral site or new phase formation.

VIII. Neutron diffraction. Cold fusion experimenters and theorists are more interested in the positions occupied by deuterons in the $\mathrm{PdD}_{\mathrm{x}}$ sublattice, as $\mathrm{x}$ approaches or exceeds unity, rather than in the relative positions of $\mathrm{Pd}$ atoms. In principle this information can be accessed directly in neutron scattering experiments. It is possible than no single experiment is of greater importance to the cold fusion community in the high-loading, heat-producing condition of $\mathrm{PdD}_{\mathrm{x} \rightarrow 1}$. An attempt to perform an in-situ electrolytic loading experiment in collaboration between SRI and Los Alamos National Laboratory, LANL, was unsuccessful for technical reasons in 1994 but further attempts should be considered. 
As the result of a large number of experiments to correlate loading and resistance ratio at SRI [12] and elsewhere [11] made mostly using methods I, IV and V, the curve originally constructed in 1990 and shown as Figure 1 was refined in 1993. Two versions of this curve were published [12-14]; these are replotted here as Figures 2 and 3. Figure 3 also took advantage of data originally published by Baranowski et. al., in 1990 [19], but comprehensively re-analyzed at SRI in 1993. These data were also included in the SRI curve (Figure 4) that sought to distinguish between successful heat producing cells (labeled in red) and unsuccessful heat producers (green) on the basis of the maximum loading or minimum resistance attained on the right side of the resistance maximum. This curve provided important confirmation of the need to measure loading and the utility of the resistance ratio method.

Absent a complete set of calibration data covering the entire range of D-Pd composition the curves in Figures 2 and 3 were constructed according to the following rules:

i) Linear multiple relationship between the $H$ and $D$ loading curves.

ii) The resistance ratio maxima for $\mathrm{H}$ and $\mathrm{D}$ occur at the same atom fraction.

iii) The high loading data for D-Pd conform with minimum standard deviation to the 1990 data of Baranowski et. al. [19].

A comprehensive review of the literature was undertaken to estimate better the position of the H-Pd resistance-loading curve and the results published as [20]. In addition a more extensive analysis was made of the Baranowski data for D-Pd published in [19]. Based on these analyses and applying rules 1-3 listed above we have constructed new curves for H-Pd and D-Pd in the high loading region. These curves are presented in Figure 5. Also plotted in Figure 5 are the equilibrium gas pressures needed to achieve the specified atomic ratios.

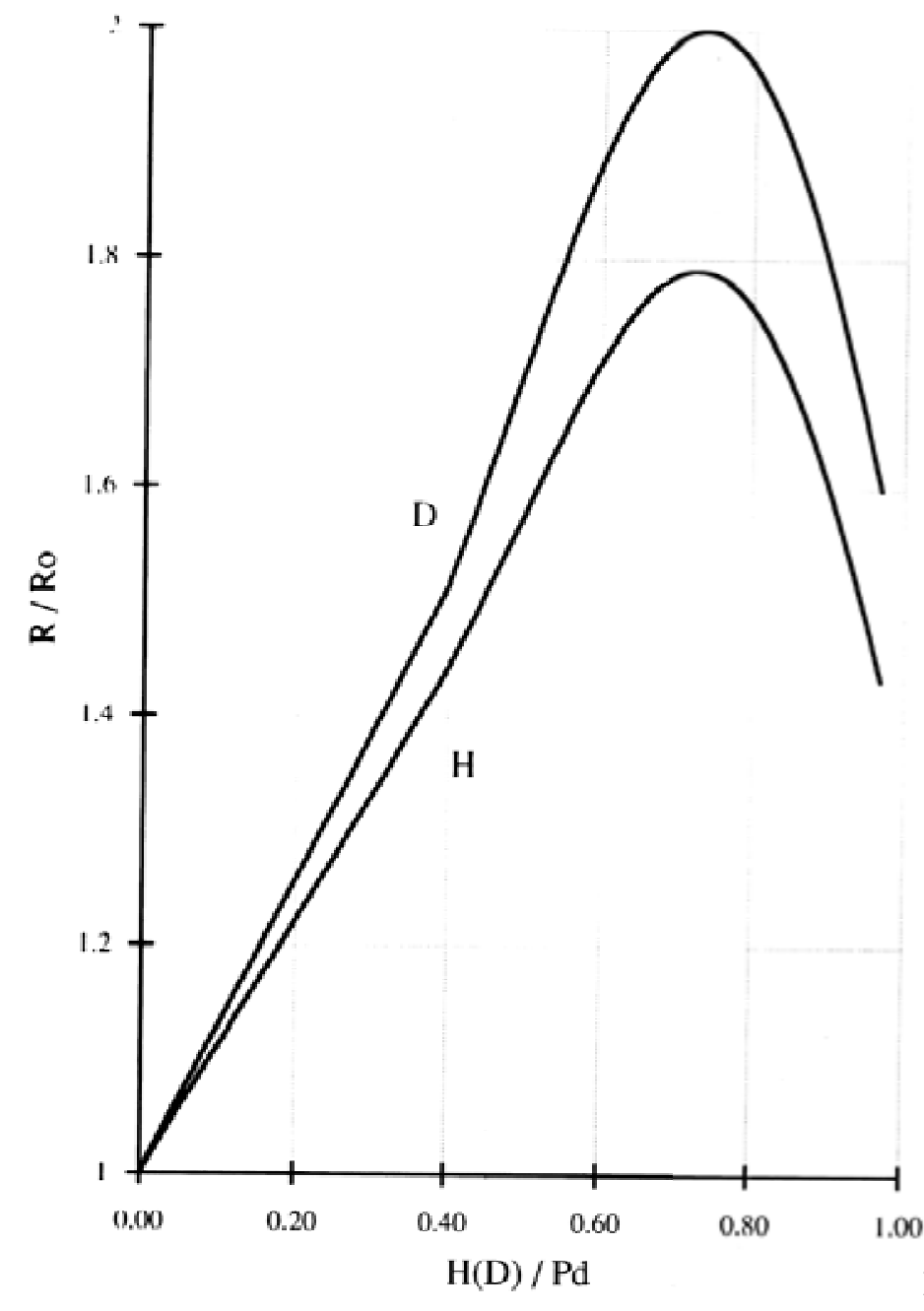

Figure 2. 


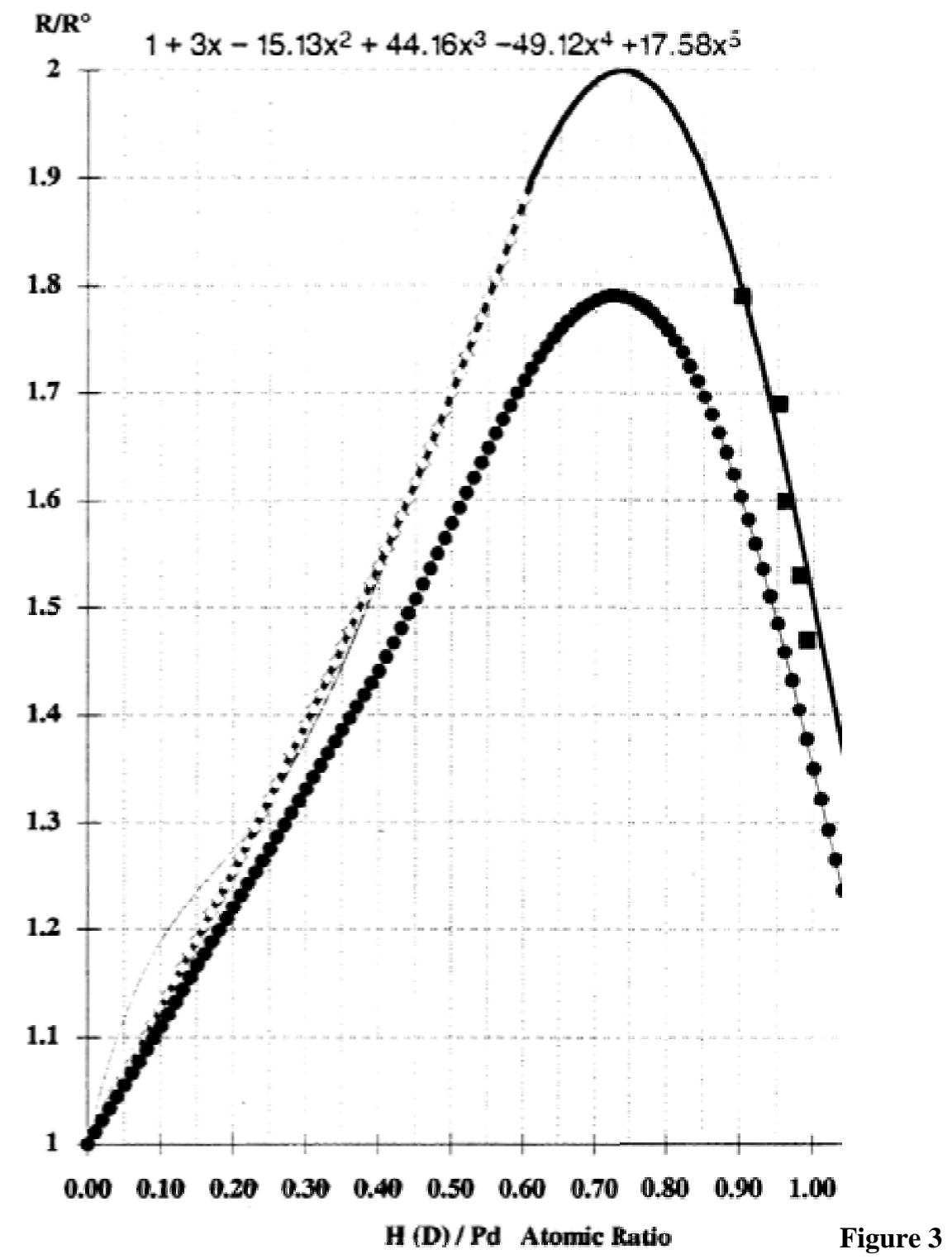

The square points in Figure 5 taken from the Baranowski data [19] exhibit an interesting kink or second order transition at $\mathrm{D} / \mathrm{Pd} \geq 1.02$. The origin of this feature is not known although it appears not to be present for $\mathrm{H}$ in the same region of composition.

Another attempt to specify the resistance/loading functions for palladium made by Zhang et. al., [21] resulted in the curves replotted in Figure 6. While the difference between the Zhang and SRI curves is subtle we believe that Zhang's curves fall outside the uncertainty in the literature data for $\mathrm{H} \mathrm{[20]} \mathrm{and} \mathrm{the}$ precision of the Baranowski data for D [19]. It is also notable, although not diagnostic, that Zhang's curves for $\mathrm{H}$ and $\mathrm{D}$ exhibit maxima at different atomic ratios (c.f. rule "2"above).

A test of accuracy of the H-Pd curve was made directly by Tripodi and the present authors [22]. The coulometric stripping method described in $\mathrm{V}$ above was used to directly correlate resistance and loading of two sections of fine Pd wire cathodes (diameter $50 \mu \mathrm{m}$, length $10 \mathrm{~cm}$ ) loaded to $\mathrm{H} / \mathrm{Pd} \approx 0.98$, sealed by codeposition of $\mathrm{Hg}[12,13]$ and then stripped at very small anodic current density. The results shown in Figure 7 do not provide a strong basis to distinguish between the Zhang and SRI curves. The knee at H/Pd $\sim 0.57$ is interesting and appears to be a real feature. Because of this knee, Zhang's attempt to accommodate data at loading $\mathrm{H} / \mathrm{Pd}<0.6$ (not attempted by SRI) appears to have moved their predicted resistance maximum to lower loading and higher maximum value. 


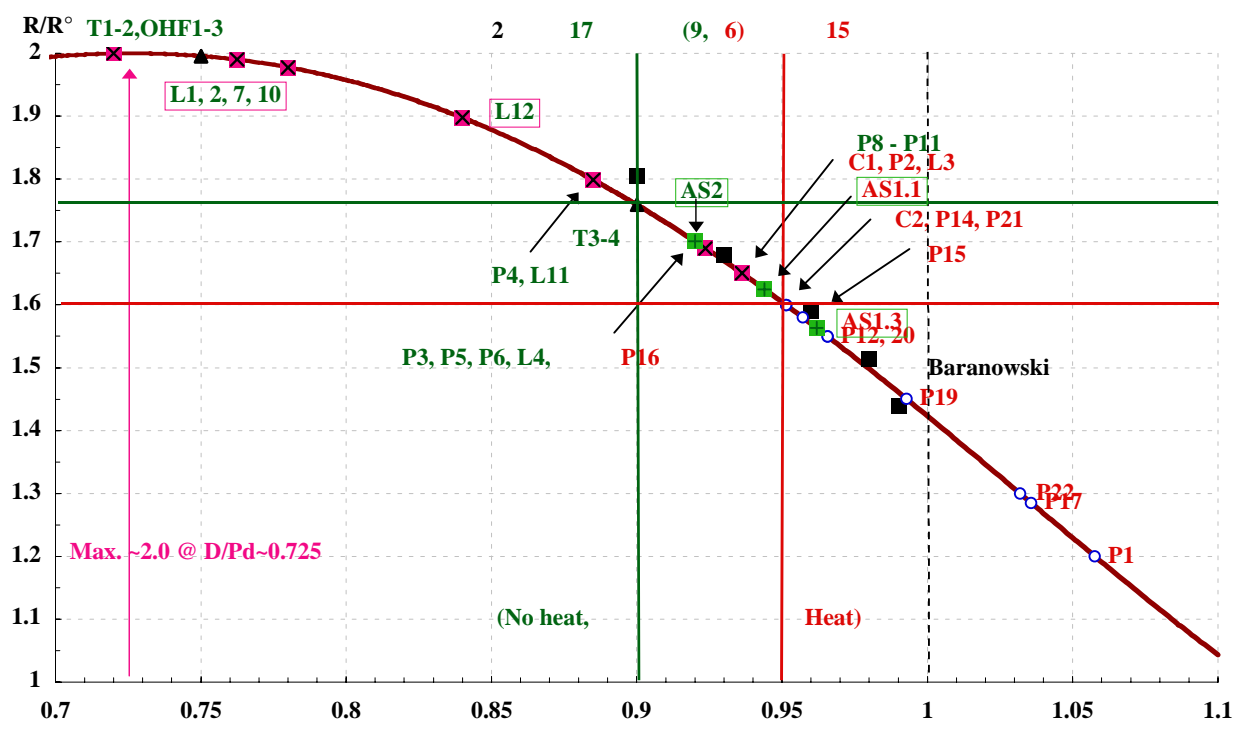

Figure 4 Showing the correlation between maximum loading measured by resistance ratio and succesful (red) and unsuccessful (green) heat production in Fleischmann-Pons electrolysis experiments performed at SRI.

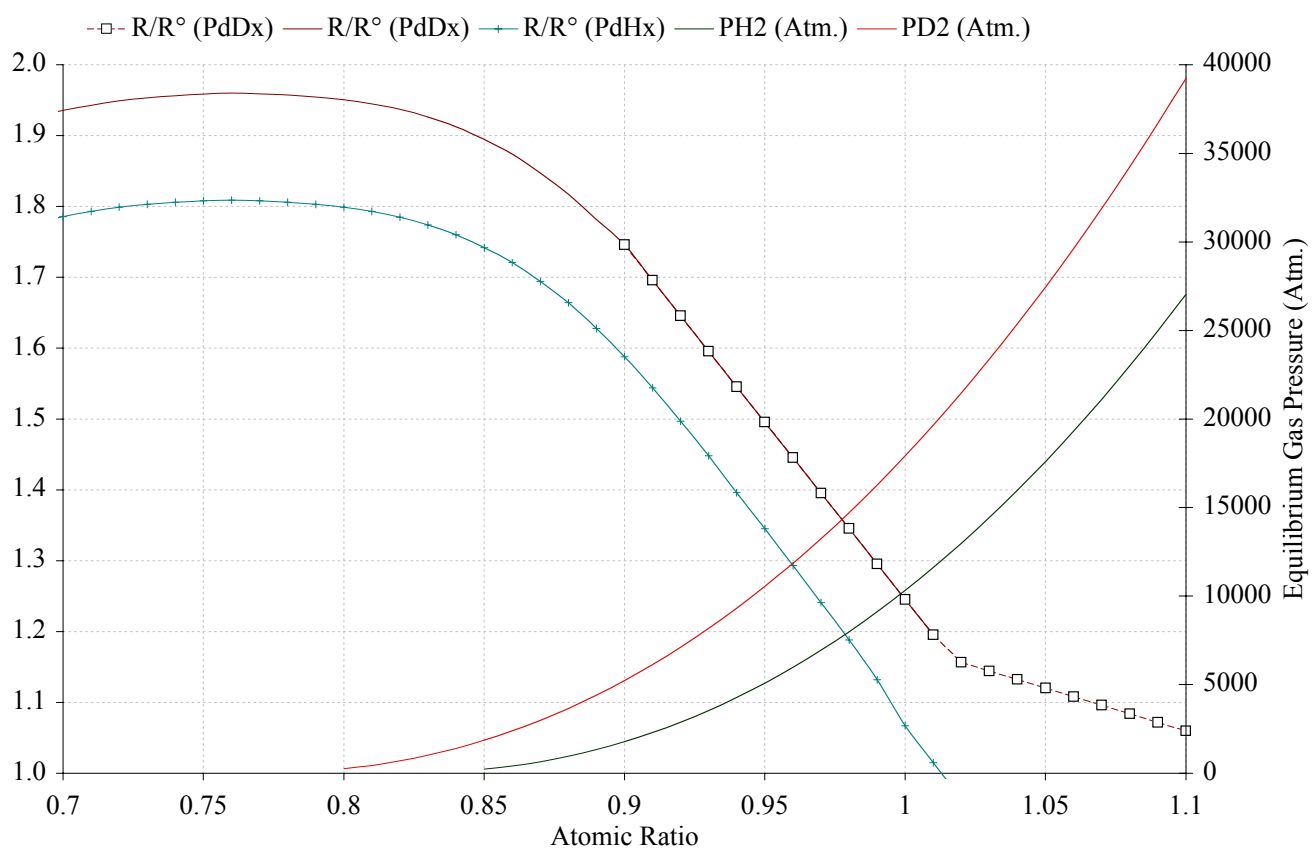

Figure 5 Improved calibration curves based on new data, reanalysis of H-Pd literature [20] and Baranowski data for D-Pd [square points 19]. 
$+\mathrm{R} / \mathrm{R}^{\circ}(\mathrm{PdHx})$ - Zhang \& Zhang H — Strip Upper — Strip Lower

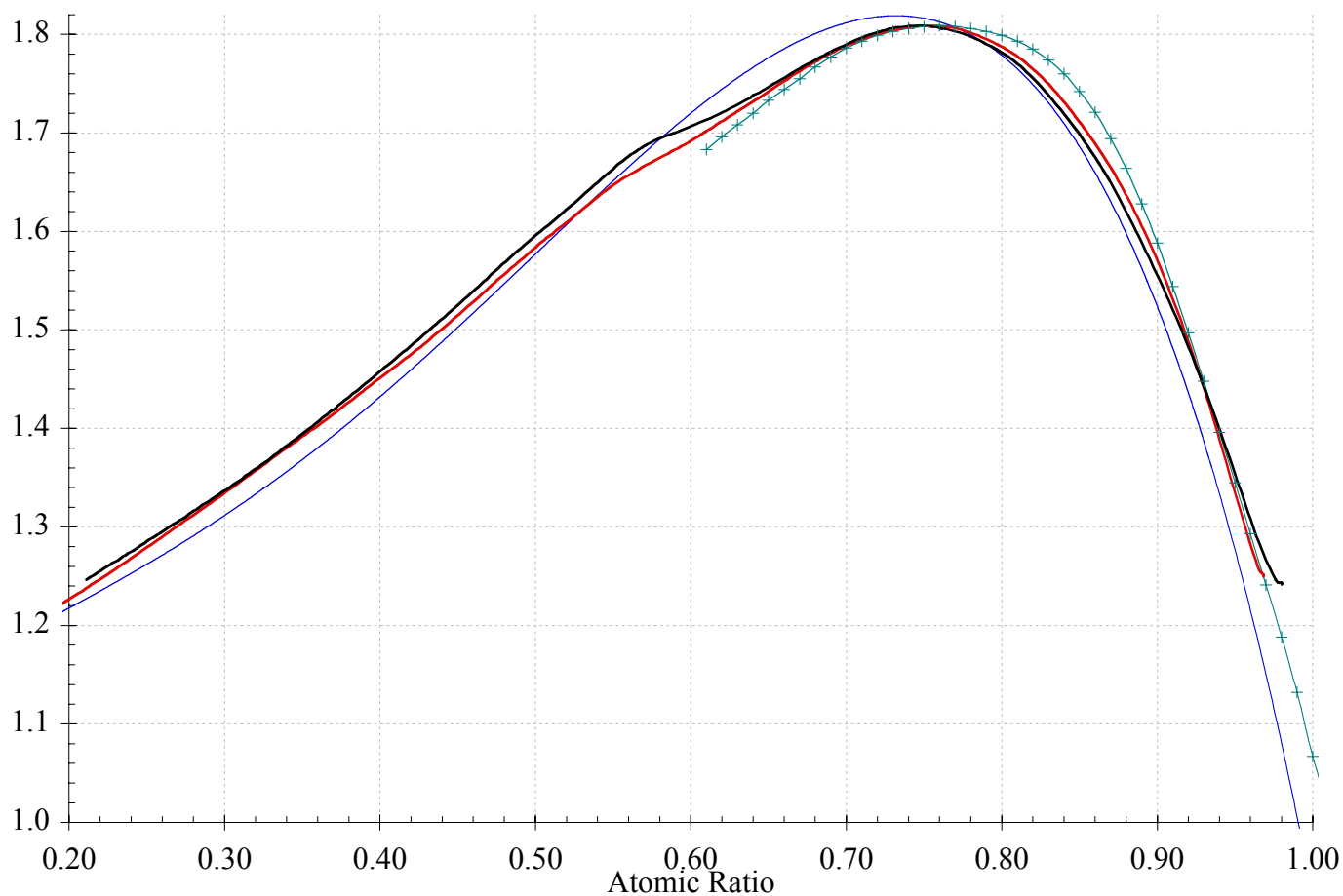

Figure 5 SRI [20] and Zhang et al [21] curves and and Tripodi et al [22] stripping data.

\section{Discussion}

The various attempts to characterize the position of the resistance versus. loading curves for $\mathrm{H}-\mathrm{Pd}$ and D-Pd can be summarized in terms of a few critical constants:

i) The maximum resistance value of the resistance ratio, $R / R^{o}{ }_{\text {Max }}$.

ii) The value of the atomic ratio at the point of maximum resistance, $X_{\text {Max }}$

iii) The value of the resistance ratio at a loading $x=1, R / R_{x=1}^{o}$.

Table 1 shows how these values have changed in 16 years of experimentation and analysis.

Table 1 Changes in Loading Parameters.

$\begin{array}{llllll} & 1990 & 1994 & 1996 & 1998 & 2002 \\ \mathrm{H} / \mathrm{Pd} & {[1]} & {[16,17,18]} & & {[20,22]} & {[21]} \\ \mathrm{R} / \mathrm{R}^{\mathrm{o}}{ }_{\text {Max }} & 1.79 & 1.78 & & 1.809 & 1.820 \\ \mathrm{X}_{\text {Max }} & 0.725 & 0.729 & & 0.760 & 0.732 \\ \mathrm{R} / \mathrm{R}_{\mathrm{x}=1}^{\mathrm{o}} & 1.34 & 1.36 & & 1.067 & 0.926 \\ & & & & & \\ \mathrm{D} / \mathrm{Pd} & & & {[19]} & {[\text { This paper] }} & \\ \mathrm{R} / \mathrm{R}^{\mathrm{o}}{ }_{\text {Max }} & 2.00 & 2.00 & 2.00 & 1.96 & 2.01 \\ \mathrm{X}_{\text {Max }} & 0.725 & 0.724 & 0.724 & 0.760 & 0.772 \\ \mathrm{R}^{\mathrm{R}} \mathrm{R}_{\mathrm{x}=1}^{\mathrm{o}} & 1.50 & 1.50 & 1.43 & 1.245 & 1.222\end{array}$


Some changes have occurred in the curve for H-Pd; a small increase in the resistance maximum and a significant increase in the position of the maximum for the SRI curve but not for Zhang's. Of far more significance to cold fusion workers the value of the resistance at $\mathrm{H} / \mathrm{Pd}=1, \mathrm{R} / \mathrm{R}_{\mathrm{x}=1}^{\mathrm{Z}}$, has dropped from $\sim 1.35$ to 1.067 for the SRI curve and below unity to 0.926 for Zhang's curve.

Turning attention to the D-Pd system the expected value of maximum resistance has changed very little with reanalysis. The position of maximum resistance in D-Pd has increased following that of H-Pd for the SRI curve using rule 2. The increase in this parameter for Zhang's curve is even larger. Of much greater significance in cold fusion studies the expected resistance at $\mathrm{D} / \mathrm{Pd}=1$ has dropped from 1.5 in 1990-1994, to 1.43 in 1996, to 1.245 in the SRI curve circa 1998. The value for Zhang calibration published in 2002 is even lower at 1.222.

The combined effect of moving the position of the resistance maximum to higher loading, and the expected resistance at $\mathrm{D} / \mathrm{Pd}=1$ to lower resistance value results in a much steeper calibration curve on the right side of the resistance maximum. As a consequence, when comparing the old calibration to the new, resistance ratio values interpreted as loadings in the region of interest for Fleischmann-Pons effect studies $(\mathrm{D} / \mathrm{Pd} \geq 0.85)$ have consistently overestimated the interstitial deuterium content of the palladium. This systematic error is not large, but it may have important physical significance.

Table 2 recalculates the maximum loading based on the minimum resistance ratio measured on the right side of the resistance maximum for five historic heat producing cells operated at SRI [12]. What had previously been interpreted as maximum average loadings at or above unity when evaluated with the new SRI calibration curve result in loadings $\mathrm{D} / \mathrm{Pd}$ approaching unity. Only P1a indicates a $\mathrm{D} / \mathrm{Pd}_{\mathrm{Max}}>1$. This cell was operated at $4^{\circ} \mathrm{C}$ where the calibration curve is likely to be different. In any case the calculated loading maximum is not outside the estimated $1 \%$ uncertainty. Thus, in experiments conducted at SRI, we have no evidence that palladium cathodes can be loaded electrolytically to atomic ratios greater than unity.

Table 2 Corrected Maximum Loading Values

$\begin{array}{llll}\text { Cell } & \mathbf{R} \mathbf{R}^{\circ}{ }_{\text {Min. }} & \text { D/Pd } \text { old } & \begin{array}{l}\text { D/Pd } \\ \text { Adjusted }\end{array} \\ \text { P1a } & 1.20 & 1.06 & 1.01 \\ \text { P17 } & 1.27 & 1.04 & 1.00 \\ \text { P22 } & 1.30 & 1.03 & 0.99 \\ \text { P19 } & 1.45 & 0.99 & 0.96 \\ \text { P12 } & 1.55 & 0.99 & 0.94\end{array}$

At this point it is worth discussing what is the relevance of a resistance ratio measurement of deuterium loading. The evidence is overwhelming that deuterium does not load homogeneously into polycrystalline palladium. Observations made of Pd cathode surfaces reveal that different crystalline facets load and expand at different rates, and the grain boundaries absorb (and release) D at very different rates from the bulk. Electronic transitions observed at low temperatures in highly loaded thin wires clearly indicate the presence of small zones of very differently (presumably higher) loaded material [14]. Hot spots observed either in-situ with thermal imaging or retrospectively by autoradiography [1], and local melting or isotopic effects, all suggest that the Fleischmann-Pons effect, whatever its cause, occurs heterogeneously throughout the bulk or on the surface. Indeed, since deuterium flux appears with equal footing to loading in quantifying excess heat power [23], the existence of heterogeneity (or time variability) in surface chemical potential appears to be crucial in producing excess heat in electrolytic D-Pd experiments.

Given these observations and the occasional solid report of excess heat at low loading one might question the deterministic power of four terminal macro-resistance ratio measurements that necessarily access a volume averaged electrode property. What might be important is simply that this property is monotonically related to the chemical potential of deuterium in the sample. The various mechanisms involved in attempts to explain the Fleischmann-Pons heat effect, the existence of a new phase or band structure, atom or ion pairing, lattice deformation to produce occupiable host sites, all depend critically on the chemical potential of absorbed deuterium. Furthermore, the very high permeability of palladium by 
deuterium ensures that adjacent regions of differing chemical potential will have only transient existence or will be associated with high deuterium fluxes, a known beneficial condition.

\section{Conclusions and Recommendations}

The calibration of the resistance ratio versus loading curve for deuterium in palladium originally published in 1990 and refined between 1992 and 1996 is in error. A re-evaluation of this curve based on literature and new experimental data indicates that the $\mathrm{D} / \mathrm{Pd}$ loading estimates in the region of interest for electrolytic Fleischmann-Pons experiments should be revised downwards. With this downward revision it is possible that electrolytic experiments performed at ambient temperature and pressure have not achieved volume averaged $\mathrm{D} / \mathrm{Pd}$ electrolytic loadings greater than unity, even in successful heat producing experiments.

In order to compare loadings obtained in different experimental setups or different laboratories it is preferable to report data as resistance ratio values, $\mathrm{R} / \mathrm{R}^{\circ}$, on the right side of the resistance maximum. In this way the shape of the resistance-loading calibration is avoided and any improvements in this function can be easily accommodated.

The issue of temperature of measurement significantly complicates the simple interpretation of a resistance ratio as an average loading, particularly in the region of interest to those studying the Fleischmann-Pons effect. The calibration curves discussed extensively here apply strictly only when $\mathrm{R}$ and $\mathrm{R}^{\circ}$ both are measured at $298 \mathrm{~K}$. The temperature coefficient of resistance is known as a function of loading, and a simple mathematical correction is often made. However, this coefficient is a strong function of composition [14] and is largely undefined for $\mathrm{PdD}_{\mathrm{x}}$ as $\mathrm{x}$ approaches and exceeds unity. Thus it is difficult to accurately apply the needed temperature corrections. For this reason it would be helpful for future workers to express clearly what, if any, temperature corrections have been made to $\mathrm{R} / \mathrm{R}^{\mathrm{o}}$, and the temperatures for which both values were measured. 


\section{References}

1. M. McKubre et al, "Calorimetry and Electrochemistry of the D-Pd System", in Proc. First Annual Cold Fusion Conference, Salt Lake City, F. Will, Ed., (1990).

2. G. Cheek and W. O'Grady, "Quartz Crystal Microbalance Study of Pd/H Interactions", ibid.

3. G. Preparata, "Theoretical Ideas on Cold Fusion", ibid.

4. P. Hagelstein"Status of Coherent Fusion Theory", ibid.

5. S. Chubb and T. Chubb, "Quantum Mechanics of 'Cold' and 'Not-So-Cold' Fusion", ibid.

6. J. Schwinger, "Nuclear Energy in an Atomic Lattice", ibid.

7. D. Macdonald, M. McKubre, A. Scott and P. Wentrcek, "Continuous In-Situ Method for the Measurement of Dissolved Hydrogen”, I\&EC Fundamantals, 20, p. 290, (1981).

8. B. Baranowski and R. Wisniewski, Phys., Stat. Sol., p. 539, (1969).

9. F. A. Lewis, "The Palladium-Hydrogen System", Academic Press, (1967).

10. E. Storms, Fusion Technol., 29, p. 261 (1996).

11. A. M. Riley et al, "Measurement of Absorption of Deuterium in Palladium During Electrolysis of Heavy Water", NCFI Final Report Vol. II, p2-123, (1991).

12. McKubre et al, EPRI TR-104195, (1993).

13. A. Spallone, F. Celani, P. Marini and V. Di Stefano, "New Electrolytic Procedure for the Obtainment of Very High H/Pd Loading Ratios", Proc. ICCF8, p191, (1990).

14. P. Tripodi, M. McKubre and F. Tanzella, "Temperature Coefficient of Resistivity at Composition Approaching PdH”, Physics Letters A, (2000).

15. G. Hubler and V. Violante personal communication (2005).

16. McKubre et al, "Loading, Calorimetric and Nuclear Investigations of the D-Pd System", Proc. ICCF4, p5-1, (1993).

17. McKubre et al, "Isothermal Flow Calorimetric Investigations of the $\mathrm{D} / \mathrm{Pd}$ and $\mathrm{H} / \mathrm{Pd}$ Systems", J. Electroanal. Chem., 368: p55, (1994).

18. McKubre et al, "Chemical and Metallurgical Issues in the Loading of D into Pd", Proc. "International Sympsium on Cold Fusion and Advanced Energy Sources", Minsk, (1994).

19. B. Baranowski, S. Filipek, M. Szustakowski, J. Farny and W. Wornya, J. Less Common Met., 158, p347, (1990).

20. S. Crouch-Baker, M. McKubre and F. Tanzella, "Variation of Resistance with composition on the ß-Phase of the H-Pd System at 298K", Z. fur Phys. Chemie., 204, p247, (1998).

21. W-S. Zhang, Z-F Zhang and Z-L Zhang, J. Electroanal. Chem., 528, p1, (2002), 571, p 81, (2004).

22. P. Tripodi, F. Tanzella and M. McKubre, unpublished results (1999).

23. McKubre et al, "Concerning Reproducibility of Excess Power...", Proc. ICCF5, p17, (1995). 